\title{
'Analytic' Wavelet Thresholding
}

\author{
By SOFIA C. OLHEDE and ANDREW T. WALDEN \\ Department of Mathematics, Imperial College London, SW7 2AZ, London, UK \\ s.olhede@imperial.ac.uk and a.walden@imperial.ac.uk.
}

\begin{abstract}
SUMMARY
We introduce so-called analytic stationary wavelet transform thresholding where, using the discrete Hilbert transform, we create a complex-valued 'analytic' vector from which an amplitude vector is defined. Thresholding of a real-valued wavelet coefficient at some transform level is carried out according to the corresponding value in this amplitude vector; relevant statistical results follow from properties of the discrete Hilbert transform. Analytic stationary wavelet transform thresholding is found to produce consistently a reduced mean squared error compared to using standard stationary wavelet transform, or 'cycle spinning,' thresholding. For signals with extensive oscillations at some transform levels, this improvement is very marked. Furthermore we show that our thresholding test is invariant to phase shifts in the data, whereas, if using complex wavelet filters, the filters must be analytic or anti-analytic at each level of the wavelet transform.

Some key words: Cycle spinning; Denoising; Hilbert transform; Phase; Thresholding; Wavelet transform.
\end{abstract}

\section{INTRODUCTION}

Donoho \& Johnstone (1994) considered the problem of estimating a signal in noise where all that is known about the signal is that it is spatially variable. They showed that wavelet-based 'universal thresholding' exhibits certain asymptotic optimality properties; see Donoho \& Johnstone (1994, p. 444) for details. Such wavelet thresholding has become a standard technique used extensively in practice and available 
in many software packages. In their model the observed $N$-length time series consists of a signal plus noise, i.e. in column vector form $x=d+\epsilon$, where $d$ is a deterministic signal of unknown regularity and $\epsilon$ represents an $N$-dimensional vector of independent and identically distributed Gaussian noise, each component being a random variable having variance $\sigma_{\epsilon}^{2}$. The discrete wavelet transform is used to transform $x$, certain subsets of coefficients are thresholded, and then the inverse transform is applied to obtain the denoised signal.

One drawback is that the coefficients are not circularly shift equivariant, so that circularly shifting the time series by some amount will not circularly shift the discrete wavelet transform coefficients by the same amount. This seriously degrades the quality of the denoising achieved. To try to alleviate this problem Coifman \& Donoho (1995) introduced the technique of 'cycle spinning;' see also Bruce et al. (1999). The idea of denoising via cycle spinning is to apply denoising not only to $x$, but also to all possible unique circularly shifted versions of $x$, and to average the results. Suppose $N$ is an integer multiple of $2^{J_{0}}$ for some integer $J_{0}$. If $\mathcal{T}^{n}$ corresponds to a circular 'delay by $n$ ' transform, and $\mathcal{T}^{-n}$ corresponds to a circular 'advance by $n$ ' transform, then, if $\widehat{d}_{n}$ is the estimator of $d$ resulting from applying a discrete wavelet transform denoising procedure to $\mathcal{T}^{n} x$, the cycle spinning denoising estimator of $d$ is given by

$$
\widehat{d}=\frac{1}{2^{J_{0}}} \sum_{n=0}^{2^{J_{0}-1}} \mathcal{T}^{-n} \widehat{d}_{n} .
$$

Shifts greater than or equal to $2^{J_{0}}$ are redundant. As pointed out by Percival \& Walden (2000, p. 429) this is equivalent to applying standard thresholding to the wavelet coefficients of the maximal overlap discrete wavelet transform, a transform we more briefly refer to as the stationary wavelet transform throughout. Some details on this transform are given in $\S 2$. In the rest of the paper we shall therefore consider that we are using the stationary wavelet transform, and it is important to recall that, for $x$ of length $N$, the transform is overdetermined and produces a zero-mean wavelet coefficient sequence $\left\{\widetilde{W}_{j, t}^{(x)}, t=0, \ldots, N-1\right\}$ at each level $j$ of the transform. Such a sequence can be written as the length- $N$ column vector 
$\widetilde{W}_{j}^{(x)}=\widetilde{\mathcal{W}}_{j} x$, where $\widetilde{\mathcal{W}}_{j}$ is the level- $j$ stationary wavelet transform matrix that $\operatorname{maps} x$ to $\widetilde{W}_{j}^{(x)}$.

Despite the appeal of stationary wavelet transform denoising, at least one aspect of the approach is unattractive, namely the thresholding of a stationary wavelet transform coefficient sequence $\left\{\widetilde{W}_{j, t}^{(x)}\right\}$ which is notably oscillatory about the mean of zero. Many physical time series give rise to oscillatory sequences of stationary wavelet transform coefficients; since the wavelet transform corresponds to a form of band-pass filtering, natural oscillatory components will manifest themselves in appropriate levels, i.e. frequency bands, and time intervals of the transform. Fig. 1(a) shows a portion of subtidal sea level measurements, in cms with a half-day sampling interval, from Crescent City, California (Percival \& Mofjeld, 1997); only frequencies lower than the lowest tidal frequency will be present. Figs 1(b)-(d) show the corresponding stationary wavelet transform coefficients for levels $j=3$ to 5 respectively.

To illustrate our approach to thresholding oscillatory sequences of stationary wavelet transform coefficients, suppose the level- $j$ coefficients for a particular signal take the form $\widetilde{W}_{j, t}^{(x)}=a_{t} \cos \left(\varphi_{t}\right), t=k / 1024, k=0, \ldots, 511$, where the amplitude is $a_{t}=\exp (-3.75 t)$ for $t \in(0.0675,0.4521)$ and zero otherwise, and $\varphi_{t}=-200 \pi t^{2}+$ $\pi / 2$; see Fig. 2(a). This is a decreasing period oscillation. Then we can write $\widetilde{W}_{j, t}^{(x)}=\Re\left\{a_{t} \exp \left(i \varphi_{t}\right)\right\}$, where $\Re$ denotes 'real part.' Small values of $\widetilde{W}_{j, t}^{(x)}$ occur regularly throughout because of the 'phase' $\varphi_{t}$, and latterly small values occur because of the decreasing amplitude $a_{t}$. With regard to thresholding we would prefer small values of the signal to be set to zero only where the amplitude is small and not also because of the phase. For the moment consider hard-thresholding this noise-free signal. With a threshold level of 0.2 , the standard approach would be to set $\widetilde{W}_{j, t}^{(x)}$ to zero if $\left|\widetilde{W}_{j, t}^{(x)}\right|$ is less than 0.2 , or otherwise to leave $\widetilde{W}_{j, t}^{(x)}$ alone. An alternative, 'amplitude-based' approach is to set $\widetilde{W}_{j, t}^{(x)}$ to zero if $a_{t}$ is less than 0.2 , or otherwise to leave $\widetilde{W}_{j, t}^{(x)}$ alone. These two thresholding approaches are illustrated in Figs 2(b) and (c). The latter approach better preserves signal continuity, but to use this approach in practice we need the amplitude $a_{t}$. 
We can define an appropriate amplitude vector by first creating a complexvalued vector $\widetilde{W}_{j}^{(x)}+i \mathcal{H} \widetilde{W}_{j}^{(x)}$, where $\mathcal{H}$ denotes the discrete Hilbert transform matrix, briefly reviewed in Appendix 1. By analogy with the theory of continuoustime signals we shall call this complex-valued vector an 'analytic' vector. The amplitudes vector then corresponds to the vector of moduli. Rather than apply $\mathcal{H}$ to $\widetilde{W}_{j}^{(x)}$ for each level of the transform in order to obtain the corresponding analytic vector, we note that $\mathcal{H}$ and $\widetilde{\mathcal{W}}_{j}$ commute, shown in $\S 3$, so that

$$
\widetilde{W}_{j}^{(x)}+i \mathcal{H} \widetilde{W}_{j}^{(x)}=\widetilde{\mathcal{W}}_{j} x+i \mathcal{H} \widetilde{\mathcal{W}}_{j} x=\widetilde{\mathcal{W}}_{j} x+i \widetilde{\mathcal{W}}_{j} \mathcal{H} x=\widetilde{\mathcal{W}}_{j} z=\widetilde{\mathcal{W}}_{j}^{(z)}
$$

say, where $z=x+i \mathcal{H} x=x+i \breve{x}$, where $\breve{x} \equiv \mathcal{H} x$. Hence, to obtain the analytic vectors $\widetilde{W}_{j}^{(x)}+i \mathcal{H} \widetilde{W}_{j}^{(x)}$ for different levels $j$ of the stationary wavelet transform, we need only calculate the analytic vector $z=x+i \mathcal{H} x$ once, and then apply the stationary wavelet transform to it in the standard way. The scaling and wavelet filters involved in $\widetilde{\mathcal{W}}_{j}$ are real-valued to avoid mixing transform and analytic vector contributions.

With $x=d+\epsilon$, note that $z$, the analytic vector corresponding to $x$ is $z=$ $d+\epsilon+i(\breve{d}+\breve{\epsilon})=(d+i \breve{d})+(\epsilon+i \breve{\epsilon})$, the sum of the analytic vector of the deterministic signal and the analytic vector of the noise. In this paper we shall apply the stationary wavelet transform to $x$ to obtain wavelet coefficients $\left\{\widetilde{W}_{j, t}^{(x)}\right\}$ at level $j$, and to the complex-valued analytic vector $z$, to obtain $\left\{\widetilde{W}_{j, t}^{(z)}\right\}$. Then thresholding of $\left\{\widetilde{W}_{j, t}^{(x)}\right\}$ will be carried out according to the size of $\left\{\left|\widetilde{W}_{j, t}^{(z)}\right|\right\}$; see Fig. 2(d).

By considering a large range of general test signals, we have found that, in using the stationary wavelet transform rather than this new proposed approach, the average estimated mean squared error is either dramatically inflated or modestly inflated. We have not encountered a situation where the proposed approach is worse than the ordinary stationary wavelet transform. 


\section{Stationary WAVELET TRANSForm AND DENOISING}

\section{$2 \cdot 1$ The stationary wavelet transform}

Given a choice of discrete wavelet transform unit-norm real-valued scaling and wavelet filters $\left\{g_{l}: l=0, \ldots, L-1\right\}$ and $\left\{h_{l}: l=0, \ldots, L-1\right\}$, first define rescaled filters, to conserve energy: $\tilde{g}_{l}=g_{l} / \sqrt{ } 2$ and $\tilde{h}_{l}=h_{l} / \sqrt{ } 2$. For the stationary wavelet transform of $x$ we set $\widetilde{V}_{0, t}^{(x)}=x_{t}, t=0, \ldots, N-1$. Then the stationary wavelet transform algorithm generates the wavelet coefficients $\left\{\widetilde{W}_{j, t}^{(x)}\right\}$ and the scaling coefficients $\left\{\widetilde{V}_{j, t}^{(x)}\right\}$ from $\left\{\widetilde{V}_{j-1, t}^{(x)}\right\}$ using the rescaled filters; the circular filterings, i.e. convolutions, can be written as

$$
\widetilde{W}_{j, t}^{(x)}=\sum_{l=0}^{L-1} \tilde{h}_{l} \widetilde{V}_{j-1,\left(t-2^{j-1} l\right) \bmod N}^{(x)} \text { and } \widetilde{V}_{j, t}^{(x)}=\sum_{l=0}^{L-1} \tilde{g}_{l} \widetilde{V}_{j-1,\left(t-2^{j-1} l\right) \bmod N}^{(x)}
$$

for $t=0, \ldots, N-1$. These coefficients can also be formulated in terms of direct circular filtering of $x$, using the level- $j$ scaling and wavelet filters $\left\{\tilde{g}_{j, l}: l=\right.$ $\left.0, \ldots, L_{j}-1\right\}$ and $\left\{\tilde{h}_{j, l}: l=0, \ldots, L_{j}-1\right\}$ of length $L_{j}=\left(2^{j}-1\right)(L-1)+1$ :

$$
\widetilde{W}_{j, t}^{(x)}=\sum_{l=0}^{L_{j}-1} \tilde{h}_{j, l} x_{(t-l) \bmod N} \text { and } \widetilde{V}_{j, t}^{(x)}=\sum_{l=0}^{L_{j}-1} \tilde{g}_{j, l} x_{(t-l) \bmod N} .
$$

Details regarding these filters may be found in Percival \& Walden (2000, Chs 4 and 5). Let $\left\{\tilde{h}_{j, l}^{\circ}\right\}$ and $\left\{\tilde{g}_{j, l}^{\circ}\right\}$ be the filters obtained by periodising $\left\{\tilde{h}_{j, l}\right\}$ and $\left\{\tilde{g}_{j, l}\right\}$ to length $N$; for example $\tilde{h}_{j, l}^{\circ}=\sum_{n=0}^{\infty} \tilde{h}_{j, l+n N}$. Then an equivalent representation is

$$
\widetilde{W}_{j, t}^{(x)}=\sum_{l=0}^{N-1} \tilde{h}_{j, l}^{\circ} x_{(t-l) \bmod N} \text { and } \widetilde{V}_{j, t}^{(x)}=\sum_{l=0}^{N-1} \tilde{g}_{j, l}^{\circ} x_{(t-l) \bmod N}
$$

At level $J_{0}$ we have $N$-length vectors $\widetilde{W}_{1}^{(x)}, \ldots, \widetilde{W}_{J_{0}}^{(x)}$ and $\widetilde{V}_{J_{0}}^{(x)}$. The stationary wavelet transform coefficient vectors can be written

$$
\widetilde{W}_{j}^{(x)}=\widetilde{\mathcal{W}}_{j} x, j=1, \ldots, J_{0} \quad \text { and } \quad \widetilde{V}_{J_{0}}^{(x)}=\widetilde{\mathcal{V}}_{J_{0}} x
$$


where $\widetilde{\mathcal{W}}_{j}$ is the $N \times N$ matrix

$$
\widetilde{\mathcal{W}_{j}}=\left[\begin{array}{cccccc}
\tilde{h}_{j, 0}^{\circ} & \tilde{h}_{j, N-1}^{\circ} & \tilde{h}_{j, N-2}^{\circ} & \cdots & \tilde{h}_{j, 2}^{\circ} & \tilde{h}_{j, 1}^{\circ} \\
\tilde{h}_{j, 1}^{\circ} & \tilde{h}_{j, 0}^{\circ} & \tilde{h}_{j, N-1}^{\circ} & \cdots & \tilde{h}_{j, 3}^{\circ} & \tilde{h}_{j, 2}^{\circ} \\
\vdots & \vdots & \vdots & \cdots & \vdots & \vdots \\
\tilde{h}_{j, N-2}^{\circ} & \tilde{h}_{j, N-3}^{\circ} & \tilde{h}_{j, N-4}^{\circ} & \cdots & \tilde{h}_{j, 0}^{\circ} & \tilde{h}_{j, N-1}^{\circ} \\
\tilde{h}_{j, N-1}^{\circ} & \tilde{h}_{j, N-2}^{\circ} & \tilde{h}_{j, N-3}^{\circ} & \cdots & \tilde{h}_{j, 1}^{\circ} & \tilde{h}_{j, 0}^{\circ}
\end{array}\right]
$$

and $\widetilde{\mathcal{V}}_{J_{0}}$ is defined similarly with $\tilde{g}_{J_{0}, l}^{\circ}$ replacing $\tilde{h}_{j, l}^{\circ}$. Note that $j=1$ corresponds to the finest resolution, and $j=J_{0}$ to the coarsest.

\section{$2 \cdot 2$ Denoising}

Coifman \& Donoho (1995) found that Stein's unbiased risk thresholding rules produced a very large number of noise spikes compared to universal thresholding when used with stationary wavelet transform/cycle spinning. They also found that 'hard thresholding and translation invariance combined gave both good visual characteristics and good quantitative characteristics'; hence we have adopted universal and hard thresholding throughout. As discussed in Percival \& Walden (2000, p. 429) cycle spinning can be implemented efficiently in terms of the stationary wavelet transform. Let $n_{j}=\widetilde{\mathcal{W}}_{j} \epsilon$. Then the algorithm consists of the following steps.

Step 1. Compute a level- $J_{0}$ partial stationary wavelet transform giving coefficient vectors $\widetilde{W}_{1}^{(x)}, \ldots, \widetilde{W}_{J_{0}}^{(x)}$ and $\widetilde{V}_{J_{0}}^{(x)}$.

Step 2. For each $j=1, \ldots, J_{0}$ apply hard thresholding using the level-dependent universal threshold with $\sigma_{n_{j}}^{2}=\sigma_{\epsilon}^{2} / 2^{j}$, to obtain

$$
\widehat{W}_{j, t}^{(x)}= \begin{cases}\widetilde{W}_{j, t}^{(x)}, & \text { if }\left|\widetilde{W}_{j, t}^{(x)}\right|>\sigma_{n_{j}} \sqrt{ }(2 \log N) \\ 0, & \text { otherwise }\end{cases}
$$

Step 3. $d$ is estimated as $\widehat{d}$ obtained by applying the inverse stationary wavelet transform to $\widehat{W}_{1}^{(x)}, \ldots, \widehat{W}_{J_{0}}^{(x)}$ and $\widetilde{V}_{J_{0}}^{(x)}$. 
If $\sigma_{\epsilon}^{2}$ is unknown, the median absolute deviation scale estimator can be adapted by using

$$
\tilde{\sigma}_{\mathrm{MAD}} \equiv \frac{2^{1 / 2} \operatorname{median}\left\{\left|\widetilde{W}_{1,0}^{(x)}\right|,\left|\widetilde{W}_{1,1}^{(x)}\right|, \ldots,\left|\widetilde{W}_{1, N-1}^{(x)}\right|\right\}}{0.6745}
$$

in place of $\sigma_{\epsilon}$.

\section{The Discrete Hilbert Transform and the Analytic Vector}

We now state three necessary lemmas, the proofs of which are given in Appendix 2.

Lemma 1. (Orthogonality.) The time series vector is orthogonal to the vector obtained by applying the transposed discrete Hilbert transform matrix to the time series, i.e.

$$
x^{\mathrm{T}} \mathcal{H}^{\mathrm{T}} x=0
$$

Lemma 2. (Commutativity.) The discrete Hilbert transform matrix $\mathcal{H}$ and the wavelet transform matrix $\widetilde{\mathcal{W}}_{j}$ commute.

Lemma 3. (Hilbert transformed noise is asymptotically uncorrelated.) Let $\epsilon$ be an $N$-dimensional vector of white noise, each random variable having mean zero and variance $\sigma_{\epsilon}^{2}$. Then asymptotically different elements of the vector $\breve{\epsilon}$ are uncorrelated and the variance of $\breve{\epsilon}_{t}$ is $\sigma_{\epsilon}^{2}$.

The commutativity of the Hilbert and stationary wavelet transforms was used in $\S 1$ to justify calculating the analytic vector once only and then applying the stationary wavelet transform. Note also that we simultaneously divide the signal into an amplitude and phase, and also its stationary wavelet coefficients. Therefore we simultaneously preserve the phase of the signal and its wavelet coefficients. The wavelet coefficients at a particular level $j$ are a projection of the signal on to the particular frequency band, and so we would expect the oscillatory nature of the signal also to be found in the projection.

While an ideal Hilbert transform should not change the covariance structure of a signal, in practice this can only be achieved with an infinite length filter, see (A1.2), and considering this the result in (A2.6) is very good. In all that follows we 
shall assume that the different elements of $\breve{\epsilon}$ are exactly uncorrelated with variance $\sigma_{\epsilon}^{2}$. With $\epsilon$ an $N$-dimensional vector of independent and identically distributed Gaussian noise in the thresholding model, it follows that $\epsilon$, and $\breve{\epsilon}$ created from it, are jointly dependent Gaussian vectors.

\section{WAVELET-TRANSFORMED NOISE AND THRESHOLDING}

\section{$4 \cdot 1$ Wavelet transform of the analytic vector}

Applying the stationary wavelet transform to $z=(d+i \breve{d})+(\epsilon+i \breve{\epsilon})$ we obtain, at level $j$,

$$
\widetilde{W}_{j}^{(z)}=\widetilde{\mathcal{W}}_{j} z=\widetilde{\mathcal{W}}_{j}(d+i \breve{d})+\widetilde{\mathcal{W}}_{j}(\epsilon+i \breve{\epsilon})
$$

If we write $s_{j}$ for the complex-valued level- $j$ stationary wavelet transform given by $\widetilde{\mathcal{W}}_{j}(d+i \breve{d})$ and let $n_{j}=\widetilde{\mathcal{W}}_{j} \epsilon$ and $\breve{n}_{j}=\widetilde{\mathcal{W}}_{j} \breve{\epsilon}$ we obtain

$$
\widetilde{W}_{j}^{(z)}=\widetilde{\mathcal{W}}_{j} z=s_{j}+\left(n_{j}+i \breve{n}_{j}\right)
$$

We now calculate the variance of $n_{j, t}$. In view of Lemma 3 the variance of $\breve{n}_{j, t}$ will be the same.

\section{$4 \cdot 2 \quad$ Noise variance}

Now $n_{j, t}=\sum_{l=0}^{N-1} \tilde{h}_{j, l}^{\circ} \epsilon_{t-l \bmod N}$, so we can write (Percival \& Walden 2000, p. 170),

$$
n_{j, t}=\frac{1}{N} \sum_{k=0}^{N-1} \widetilde{H}_{j}\left(\frac{k}{N}\right) \Xi_{k} e^{i 2 \pi t k / N},
$$

where $\widetilde{H}_{j}(f)=\sum_{l=0}^{L_{j}-1} \tilde{h}_{j, l} e^{-i 2 \pi l f}$ and $\Xi_{k}=\sum_{t=0}^{N-1} \epsilon_{t} e^{-i 2 \pi t k / N}$. Then, since the realvalued noise $n_{j, t}$ has mean zero,

$$
\operatorname{var}\left(n_{j, t}\right)=E\left(n_{j, t} n_{j, t}^{*}\right)=E\left\{\frac{1}{N^{2}} \sum_{k=0}^{N-1} \sum_{l=0}^{N-1} \widetilde{H}_{j}\left(\frac{k}{N}\right) \widetilde{H}_{j}^{*}\left(\frac{l}{N}\right) \Xi_{k} \Xi_{l}^{*} e^{i 2 \pi t(k-l) / N}\right\} .
$$

Let $\operatorname{var}\left(n_{j, t}\right)=\sigma_{n_{j}}^{2}$, say. From (A2.5) we know that $E\left\{\Xi_{k} \Xi_{l}^{*}\right\}=N \sigma_{\epsilon}^{2} \delta_{k, l}$, and so

$$
\begin{aligned}
\sigma_{n_{j}}^{2} & =\frac{\sigma_{\epsilon}^{2}}{N} \sum_{k=0}^{N-1} \sum_{l=0}^{N-1} \widetilde{H}_{j}\left(\frac{k}{N}\right) \widetilde{H}_{j}^{*}\left(\frac{l}{N}\right) \delta_{k, l} e^{i 2 \pi t(t-l) / N} \\
& =\frac{\sigma_{\epsilon}^{2}}{N} \sum_{k=0}^{N-1}\left|\widetilde{H}_{j}\left(\frac{k}{N}\right)\right|^{2}=\sigma_{\epsilon}^{2} \sum_{l=0}^{N-1}\left[\tilde{h}_{j, l}^{\circ}\right]^{2}
\end{aligned}
$$


The length of the wavelet filter at level $j$ is $L_{j}=\left(2^{j}-1\right)(L-1)+1$. Define $j_{p}=\max \left\{j: L_{j} \leq N\right\}$; that is $j_{p}$ is the largest value $j$ such that the filter is not periodised. Then, if $j \leq j_{p}$, the filter $\tilde{h}_{j, l}^{\circ}$ is identical to $\tilde{h}_{j, l}$ for which we know by design that $\sum_{l=0}^{N-1} \tilde{h}_{j, l}^{2}=2^{-j}$. Hence

$$
\sigma_{n_{j}}^{2}=\sigma_{\epsilon}^{2} \sum_{l=0}^{N-1}\left(\tilde{h}_{j, l}^{\circ}\right)^{2}=\sigma_{\epsilon}^{2} / 2^{j} .
$$

In our examples in $\S 4.5, J_{0}=j_{p}$, so that (4.2) always holds; if however levels $j>j_{p}$ were used then length restrictions apply to the time series in order to ensure that (4.2) holds (Walden \& Contreras Cristán, 1998, Theorem 1).

\section{$4 \cdot 3$ Statistical properties of the real and imaginary parts}

We shall show that coincident elements of $n_{j}$ and $\breve{n}_{j}$ are independent. Since the elements are zero-mean Gaussian we need merely to show that, for all $0 \leq t \leq$ $N-1$

$$
\left\{E\left(n_{j} \breve{n}_{j}^{\mathrm{T}}\right)\right\}_{t t}=0
$$

that is, all diagonal elements of the covariance matrix are zero. Now

$$
\begin{aligned}
E\left(n_{j} \breve{n}_{j}^{\mathrm{T}}\right) & =E\left(\widetilde{\mathcal{W}}_{j} \epsilon \breve{\epsilon}^{\mathrm{T}} \widetilde{\mathcal{W}}_{j}^{\mathrm{T}}\right)=E\left\{\widetilde{\mathcal{W}}_{j} \epsilon(\mathcal{H} \epsilon)^{\mathrm{T}} \widetilde{\mathcal{W}}_{j}^{\mathrm{T}}\right\}=\widetilde{\mathcal{W}}_{j} E\left(\epsilon \epsilon^{\mathrm{T}}\right) \mathcal{H}^{\mathrm{T}} \widetilde{\mathcal{W}}_{j}^{\mathrm{T}} \\
& =\sigma_{\epsilon}^{2} \widetilde{\mathcal{W}}_{j} \mathcal{H}^{\mathrm{T}} \widetilde{\mathcal{W}_{j}^{\mathrm{T}}}=\sigma_{\epsilon}^{2}\left(\begin{array}{c}
u_{1}^{\mathrm{T}} \\
\vdots \\
u_{N}^{\mathrm{T}}
\end{array}\right) \mathcal{H}^{\mathrm{T}}\left(u_{1}, \ldots, u_{N}\right)
\end{aligned}
$$

say, where $u_{i}$ is the $i$ th column vector of $\widetilde{\mathcal{W}}_{j}^{\mathrm{T}}$. Then, using (3.1), we have

$$
\left\{E\left(n_{j} \breve{n}_{j}^{\mathrm{T}}\right)\right\}_{t t}=\sigma_{\epsilon}^{2} u_{t}^{\mathrm{T}} \mathcal{H}^{\mathrm{T}} u_{t}=0
$$

Hence at any time point $t$, the random variables $n_{j, t}$ and $\breve{n}_{j, t}$ are uncorrelated, but the vectors $n_{j}$ and $\breve{n}_{j}$ are not uncorrelated in general. However, since $n_{j}$ is dependent Gaussian, $n_{j}$ and $\breve{n}_{j}$ are jointly dependent Gaussian and the random variables $n_{j, t}$ and $\breve{n}_{j, t}$ are independent and identically distributed for any specified $t$, with mean zero and variance $\sigma_{n_{j}}$, say. 
Let us write the $t$ th element of $\widetilde{W}_{j}^{(z)}$ in (4.1) as

$$
\widetilde{W}_{j, t}^{(z)}=s_{j, t}+\left(n_{j, t}+i \breve{n}_{j, t}\right)=s_{j, t}+\sigma_{n_{j}}\left(\eta_{j, t}+i \zeta_{j, t}\right), t=0, \ldots, N-1
$$

where $\eta_{j, t}$ and $\zeta_{j, t}$ are independent, zero mean and unit variance Gaussian random variables. Then

$$
\left(\eta_{j, 0}, \zeta_{j, 0}, \ldots, \eta_{j, N-1}, \zeta_{j, N-1}\right)^{\mathrm{T}}
$$

has a multivariate Gaussian distribution with mean zero and covariance matrix

$$
\Sigma=\left(\begin{array}{cccc}
I_{11} & \Sigma_{12} & \ldots & \Sigma_{1 N} \\
\Sigma_{21} & I_{22} & \ldots & \vdots \\
\vdots & \vdots & \ddots & \vdots \\
\Sigma_{N 1} & \ldots & \ldots & I_{N N}
\end{array}\right)
$$

where $I_{k k}$ is a $2 \times 2$ identity matrix and $\Sigma_{k l}$ is an unspecified component of the covariance matrix.

\subsection{Thresholds}

Given the covariance matrix $\Sigma$ of (4.3), the following inequality follows from Corollary 2 of Dykstra (1980),

$$
\operatorname{pr}\left\{\bigcap_{t=0}^{N-1}\left(\eta_{j, t}^{2}+\zeta_{j, t}^{2} \leq \delta^{2}\right)\right\} \geq \prod_{t=0}^{N-1} \operatorname{pr}\left(\eta_{j, t}^{2}+\zeta_{j, t}^{2} \leq \delta^{2}\right)=\left(1-e^{-\frac{1}{2} \delta^{2}}\right)^{N} .
$$

The last term follows because $\eta_{j, t}^{2}+\zeta_{j, t}^{2} \sim \chi_{2}^{2}$. If we define $M_{N}^{2}=\max _{t=0, \ldots, N-1}\left\{\eta_{j, t}^{2}+\right.$ $\left.\zeta_{j, t}^{2}\right\}$, then $\operatorname{pr}\left(M_{N}^{2} \leq \delta^{2}\right)=\operatorname{pr}\left\{\bigcap_{t=0}^{N-1}\left(\eta_{j, t}^{2}+\zeta_{j, t}^{2} \leq \delta^{2}\right)\right\}$, while, if we let $C_{N}^{2}=$ $\max _{t=0, \ldots, N-1}\left\{\nu_{t}\right\}$, where $\left\{\nu_{t}\right\}$ are independent $\chi_{2}^{2}$ variables, then $\operatorname{pr}\left(C_{N}^{2} \leq \delta^{2}\right)=$ $\left(1-e^{-\frac{1}{2} \delta^{2}}\right)^{N}$. Therefore, if $\lim _{N \rightarrow \infty} \operatorname{pr}\left(C_{N}^{2}>\delta^{2}\right)=p$ then (4.4) tells us that $\lim _{N \rightarrow \infty} \operatorname{pr}\left(M_{N}^{2}>\delta^{2}\right) \leq p$.

Hence thresholds based on independent $\chi_{2}^{2}$ variables, studied by Sardy (2000), will be conservative for our application. Sardy suggests taking $\delta^{2}=2 \log \{N \log (N)\}$, from which we note that

$$
\operatorname{pr}\left(C_{N}^{2} \leq \delta^{2}\right)=\left(1-e^{-\frac{1}{2} \delta^{2}}\right)^{N}=\left\{1-\frac{1}{N \log (N)}\right\}^{N} \rightarrow 1,
$$


as $N \rightarrow \infty$.

Thresholding proceeds under the hypothesis of no deterministic signal being present; in this case

$$
\widetilde{W}_{j, t}^{(z)}=\sigma_{n_{j}}\left(\eta_{j, t}+i \zeta_{j, t}\right), t=0, \ldots, N-1,
$$

and the hard-thresholding rule, combined with Sardy's threshold level, gives

$$
\widehat{W}_{j, t}^{(x)}= \begin{cases}\widetilde{W}_{j, t}^{(x)}, & \text { if }\left|\widetilde{W}_{j, t}^{(z)}\right|>\sigma_{n_{j}} \sqrt{ }\{2 \log (N \log N)\} \\ 0, & \text { otherwise }\end{cases}
$$

for levels $j=1, \ldots, J_{0}$. Hence our new algorithm, 'analytic stationary wavelet transform thresholding,' consists of Steps 1 to 3 of the algorithm of $\S 2 \cdot 2$, but with (2.2) replaced by (4.5).

\subsection{Examples}

We use throughout the 'least asymmetric' Daubechies filter of width $L=8$. The examples comprise five synthetic signals and two recorded signals. The first six use $N=1024$ and the stationary wavelet transform is carried out to level $J_{0}=7$ so that, as is common practice, low frequencies, here $f \in[0,1 / 256]$, are not subjected to thresholding. The last has $N=2048$ and $J_{0}=8$. The synthetic series are generated according to $x_{t}=d_{t}+\epsilon_{t}, t=0, \ldots, N-1$, where $\epsilon$ is an $N$-dimensional vector of independent and identically distributed Gaussian noise. We took a signal variance to noise variance ratio, i.e., signal-to-noise variance ratio, of 50. Note that this is almost identical to the standard deviation ratio of 7 used in Donoho \& Johnstone (1994). We also considered the lower signal-to-noise variance ratio of 10, corresponding to increased noise. The models for $d_{t}$ take the following forms.

Model 1. A cosine with decreasing amplitude, $d_{t}=\exp (-3 t / N) \cos (0.2 \pi t-\pi / 2)$.

Model 2. Donoho \& Johnstone's Doppler,

$$
d_{t}=\left\{\frac{t}{N}\left(1-\frac{t}{N}\right)\right\}^{1 / 2} \sin \left(2 \pi \frac{1.05}{\frac{t}{N}+0.05}\right) .
$$


Model 3. An increasing frequency 'chirp' signal, $d_{t}=\sin \left\{\pi\left(t+\frac{N}{2}\right)^{2} / 8 N\right\}$.

Model 4. A sinusoid with a gap, $d_{t}=\sin (0.1 \pi t)$, for $t=0, \ldots, 449$ and $t=$ $574, \ldots, 1023$, and $d_{t}=0$, for $t=450, \ldots, 573$.

Model 5. The 'blocks' function of Donoho \& Johnstone (1994).

Model 6. The portion of subtidal sea level measurements shown in Fig. 1.

Model \%. The radial component of the 1991 Solomon Islands earthquake in 'instrument counts' with a 2s sampling interval; this series is shown in Lilly \& Park (1995).

For both the recorded signals the level $j=1$ stationary wavelet transform sequence was zeroed and the signal reconstructed. Noise was then added. This was done to add integrity to the simulations from the real signals since $\tilde{\sigma}_{\mathrm{MAD}}$, the estimate of $\sigma_{\epsilon}$, is computed from the level 1 coefficients, assumed to be mostly noise, as is the case for the synthetic signals.

We define the estimated mean squared error for simulation run $k$ as $\alpha_{k}=$ $\frac{1}{N} \sum_{t=0}^{N-1}\left(d_{t}-\widehat{d}_{t, k}\right)^{2}$ where $\widehat{d}_{t, k}$ is the estimate of $d_{t}$ for simulation run $k$. We can then calculate the average $\bar{\alpha}$ of the $\alpha_{1}, \ldots, \alpha_{n}$ over $n$ simulation runs. Denoting $\bar{\alpha}$ by $\bar{\alpha}_{\mathrm{M}}$ when using standard stationary wavelet transform thresholding and by $\bar{\alpha}_{\mathrm{A}}$ when using analytic stationary wavelet transform thresholding, we can for each example define the percentage average estimated mean squared error inflation when using stationary wavelet transform rather than analytic stationary wavelet transform thresholding as

$$
\bar{\alpha}_{p}=\frac{\bar{\alpha}_{\mathrm{M}}-\bar{\alpha}_{\mathrm{A}}}{\bar{\alpha}_{\mathrm{A}}} \times 100 .
$$

Values of $\bar{\alpha}_{p}$ are given in Table 1 for $n=100$ simulation runs. We can see that analytic stationary wavelet transform thresholding offers either modest or large improvements over the stationary wavelet transform. By way of example, Fig. 3 gives a magnified view of part of the denoised Doppler signal using the analytic stationary wavelet transform and the standard stationary wavelet transform. 
Model 5 was included as a basically non-oscillatory signal with isolated discontinuities at which our method is not targeted. Nevertheless the results are good, and show a modest improvement over the basic stationary wavelet transform.

The more extensive and stronger the oscillations, so that the threshold level is exceeded, the bigger the advantage, but the important point is that a worthwhile improvement has always been found to accrue.

\section{5. 'PHASE-ShIFT' INVARIANCE}

\section{$5 \cdot 1 \quad$ A theorem}

As noted above, real wavelet analysis is very sensitive to both time and phase alignment. Cycle spinning takes care of time alignments, but what of the common physical problem of phase shifts (White, 1988; Walden \& Williams, 1993)? Fig. 4(a) shows Donoho \& Johnstone's Doppler signal (4.6) and a $\phi=\pi / 4$ phaseshifted version of it.

Magnitude, i.e. amplitude, and phase require two observations at any timepoint. In our analytic vector approach we made use of complex wavelet coefficients, as they have the notion of amplitude and phase clearly defined, unlike real coefficients. We might instead have used complex-valued wavelet filters along with the real data to produce complex wavelet coefficients. We examine these two alternatives with respect to phase shifts.

Theorem 1. We assume that the wavelet filters used have no energy at zero frequency and that the data have no energy at the Nyquist frequency.

(i) Consider first using real-valued wavelet and scaling filters. Then the magnitudes of the level-j stationary wavelet coefficients of the analytic vector are invariant to a phase shift in the signal.

(ii) Consider secondly using complex-valued wavelet and scaling filters. Then a necessary condition for the magnitudes of the level-j stationary wavelet coefficients of the data vector to be invariant to a phase shift in the signal is 
that the frequency responses of the real and imaginary parts of the level-j complex-valued filters must have identical magnitudes and differ in phase by $\pm \pi / 2$ for Fourier frequencies $k / N, k \neq(N / 2)$.

Proof. This may be found in Appendix 2.

Corollary 1. From the first part of the theorem it follows that our thresholding test, $\left|\widetilde{W}_{j, t}^{(z)}\right|>\sigma_{n_{j}} \sqrt{ }\{2 \log (N \log N)\}$, is invariant to phase shifts.

Corollary 2. From the second part of the theorem, a necessary condition for the invariance of wavelet coefficient magnitudes to phase shifts is that the level-j complex-valued filters are analytic or anti-analytic.

Unfortunately we cannot simply create analytic or anti-analytic wavelet filters via the discrete Hilbert transform since the circularity would be catastrophic to the required wavelet filter properties. It would be necessary to use the infinite impulse response (A1.2), and then the filters would not have finite support.

We now illustrate the theorem using the Doppler signal example and some standard real and complex wavelet filters; the assumptions of the theorem are satisfied.

\section{$5 \cdot 2$ Analytic vector and real-valued wavelet filters}

We here illustrate Corollary 1 . We start with the vector $x$ and, as in $\S 1$, calculate the complex-valued analytic vector $x+i \mathcal{H} x$ and then apply the stationary wavelet transform using real-valued wavelet coefficients. Amplitude and phase vectors are defined in the obvious way as the magnitude and arg of the analytic vector. The solid lines in Fig. 4(b) give the magnitudes of the stationary wavelet transform coefficients for levels 1 to 3 when using Daubechies extremal phase filters of length ten. Also shown dotted is the same quantity starting instead with the $\pi / 4$ phaseshifted version; these dotted lines cannot be perceived as they are totally overlain by the solid lines, since the coefficient magnitudes are invariant to the phase-shift. 


\section{$5 \cdot 3$ Real vector and complex-valued wavelet filters}

We now turn to Corollary 2. The real data vector is subjected to the stationary wavelet transform using complex-valued scaling and wavelet filters. The solid lines in Fig. 4(c) give the magnitudes of the stationary wavelet transform coefficients for levels 1 to 3 when we use a complex Daubechies wavelet filter of length ten (Lina \& Mayrand, 1995). As can be seen in Fig. 4(c) the complex filter does not provide invariance to phase shifts. From Fig. 4(d) we see that even at level $j=1$ the real and imaginary parts have pass-bands of different magnitudes in different parts of the frequency domain, whereas we know from Theorem 1 that these must be identical. Note also that to obtain a chi-squared distribution for the squared amplitude it is necessary for the real and imaginary part of the filters to have the same norm. A similar result holds for the length-six Lawton wavelet (Lawton, 1993) which was shown to perform well for complex denoising by Stuart Barber and Guy Nason in a currently unpublished paper entitled 'Real nonparametric regression using complex wavelets.'

If we use these complex filters, a constant phase-shift of the data changes the magnitude of the wavelet coefficients at any $(j, t)$ doublet. The magnitude of the wavelet coefficient at a $(j, t)$ does not provide a sensible division into 'amplitude' and 'phase', and absorbs some of the oscillatory properties of the 'phase' and so the amplitude is oscillatory, as is evident from Fig. 4(c). In their paper Barber and Nason noted that there seemed to be no reason to preserve the phase of the wavelet coefficients they found using the complex Daubechies wavelets, and our results support and amplify this assertion.

\section{Computational Overhead}

The only overheads in using the analytic stationary wavelet transform thresholding are (i) the computation of the discrete Hilbert transform of the observed data vector, and (ii) computation of the stationary wavelet transform of the complex analytic vector in addition to the stationary wavelet transform of the observed 
data vector. The analytic vector computation can be carried out using the fast Fourier transform and is given in Appendix 1, (A1.4). Both (i) and (ii) thus involve $O\left(N \log _{2} N\right)$ algorithms which make the method very efficient in practice.

\section{ACKNOWLEDGEMENT}

This work was begun when Sofia Olhede was Beit Scientific Research Fellow, recipient of a grant from the Engineering and Physical Science Research Council, and beneficiary of the Helge Ax:son Johnsons Stiftelse. We thank Hal Mofjeld and Don Percival for the sea level data, Jeff Park for the earthquake data and the Editor and referees for their constructive comments and suggestions which led to a much improved exposition.

\section{APPENDIX 1}

\section{The Hilbert transform}

Continuous time and continuous frequency. The Hilbert transform of a real-valued finite-energy function $x(t)$ is the real-valued function $\breve{x}(t)$ given by (Papoulis, 1991)

$$
\breve{x}(t)=x(t) * \frac{1}{\pi t}=\frac{1}{\pi} \int_{-\infty}^{\infty} \frac{x(u)}{t-u} d u,
$$

where $*$ denotes convolution, so that $\breve{x}(t)$ is seen to be the convolution of $x(t)$ and $(\pi t)^{-1}$, and, since when $t=u$ the integral diverges, it is defined in terms of the Cauchy principal value.

The Fourier transform $G(f)$ of $(\pi t)^{-1}$ is given by

$$
G(f)=-i \operatorname{sgn}(f)= \begin{cases}-i, & \text { if } f>0 \\ 0, & \text { if } f=0 \\ i, & \text { if } f<0\end{cases}
$$

The convolution of $x(t)$ with $(\pi t)^{-1}$ is equivalent to filtering $x(t)$. The transfer function, or frequency response function, of the filter is $G(f)$ and $G(f)=$ $|G(f)| \exp \{i \theta(f)\}$, where $|G(\cdot)|$ and $\theta(\cdot)$ are called, respectively, the gain function 
and phase function of the filter. We see that $G(f)$ is totally imaginary, so that $|G(f)|=1$ for all $f$, and

$$
\theta(f)= \begin{cases}-\pi / 2, & \text { if } f>0 \\ 0, & \text { if } f=0 \\ \pi / 2, & \text { if } f<0\end{cases}
$$

Thus Hilbert transforming is equivalent to leaving the energy spectrum of the signal unchanged while changing the phase by $-\pi / 2$ for positive frequencies and $\pi / 2$ for negative frequencies.

Fourier transformation of the complex-valued signal $z(t)=x(t)+i \breve{x}(t)$ gives

$$
Z(f)=X(f)+i\{-i \operatorname{sgn}(f)\} X(f)= \begin{cases}2 X(f), & \text { if } f>0, \\ X(f), & \text { if } f=0 \\ 0, & \text { if } f<0 .\end{cases}
$$

Consequently, since $Z(f)$ is zero for negative frequencies,

$$
z(t)=\int_{0}^{\infty} Z(f) e^{i 2 \pi f t} d f
$$

Since $f$ is never negative under the integral, $t$ can be replaced by $\tau=t+i u$, with $u>0$, and the integral is still convergent. Then

$$
z(\tau)=\int_{0}^{\infty} Z(f) e^{i 2 \pi f \tau} d f, \quad \tau=t+i u, u>0
$$

is an analytic function over the upper half-plane and $z(t)$ is called the analytic signal.

Discrete time and continuous frequency. The infinite length impulse response sequence or digital filter corresponding to the frequency response $-i \operatorname{sgn}(f)$ is (Oppenheim \& Schafer, 1975, p. 360)

$$
q_{l}=\int_{-1 / 2}^{1 / 2}-i \operatorname{sgn}(f) e^{i 2 \pi f l} d f= \begin{cases}\frac{2 \sin ^{2}(l \pi / 2)}{l \pi}, & \text { if } l \text { odd } \\ 0, & \text { if } l \text { even }\end{cases}
$$


Discrete time and discrete frequency. Assume that $N$ is even. With reference to the Fourier frequencies $f_{k}=k / N$, we define a discrete sign sequence via

$$
\operatorname{sgnd}_{k}= \begin{cases}0, & k=0 \\ 1, & k=1, \ldots, \frac{N}{2}-1 \\ 0, & k=\frac{N}{2} \\ -1, & k=\frac{N}{2}+1, \ldots, N-1 .\end{cases}
$$

Then given the vector $x^{\mathrm{T}}=\left(x_{0}, \ldots, x_{N-1}\right)$ with discrete Fourier transform

$$
X_{k}=\sum_{t=0}^{N-1} x_{t} e^{-i 2 \pi t k / N}, \quad k=0, \ldots, N-1,
$$

the equivalent of (A1.1) is

$$
Z_{k}=X_{k}+i\left(-i \operatorname{sgnd}_{k}\right) X_{k}= \begin{cases}X_{0}, & k=0 \\ 2 X_{k}, & k=1, \ldots, \frac{N}{2}-1 \\ X_{\frac{N}{2}}, & k=\frac{N}{2} \\ 0, & k=\frac{N}{2}+1, \ldots, N-1 .\end{cases}
$$

The elements of the 'analytic' vector are found via the inverse discrete Fourier transform of $\left\{Z_{k}\right\}$ :

$$
z_{t}=\frac{1}{N} \sum_{k=0}^{N-1} Z_{k} e^{i 2 \pi k t / N}, \quad t=0, \ldots, N-1 .
$$

The inverse discrete Fourier transform of the frequency response sequence $-i \operatorname{sgnd}_{k}, k=0, \ldots, N-1$, namely

$$
q_{l}^{\circ}=-\frac{1}{N} \sum_{k=0}^{N-1} i \operatorname{sgnd}_{k} e^{i 2 \pi k l / N}, \quad l=0, \ldots, N-1,
$$

identifies the filter $q_{l}^{\circ}, l=0, \ldots, N-1$, which is a periodisation of $\left\{q_{l}\right\}$ in (A1.2); that is, $q_{l}^{\circ}=\sum_{n=-\infty}^{\infty} q_{l+n N}$, where (A1.5) may be written as in (A2.2). Thus $x_{t}=\Re\left\{z_{t}\right\}$ and $\breve{x}_{t}=\Im\left\{z_{t}\right\}$ with $\breve{x}_{t}$ given by (A2.1). With $\left\{z_{t}\right\}$ defined as in (A1.4) we also know (Marple, 1999, p. 2601) that the real and imaginary parts of $z$ are orthogonal; see (A2.4). 


\section{APPENDIX 2}

\section{Proofs}

Proof of Lemma 1. The discrete Hilbert transform is derived and discussed in Appendix 1, where the results (A2.1), (A2.2) and (A2.4) quoted below are justified. Given a vector $x$ with elements $\left\{x_{t}\right\}$, the elements $\left\{\breve{x}_{t}\right\}$ of the vector $\breve{x}$ of its discrete Hilbert transform are given by

$$
\breve{x}_{t}=\sum_{l=0}^{N-1} q_{l}^{\circ} x_{(t-l) \bmod N}, \quad t=0, \ldots, N-1,
$$

where the periodised filter $q_{l}^{\circ}$ is given by

$$
q_{l}^{\circ}= \begin{cases}(2 / N) \cot (l \pi / N), & l \text { odd } \\ 0, & l \text { even }\end{cases}
$$

for $l=0, \ldots, N-1$. We can write the discrete Hilbert transform in matrix form as $\breve{x}=\mathcal{H} x$ where, because of (A2.1) and (A2.2),

$$
\mathcal{H}=\left[\begin{array}{rrrrrrrr}
0 & q_{N-1}^{\circ} & 0 & q_{N-3}^{\circ} & 0 & \ldots & 0 & q_{1}^{\circ} \\
q_{1}^{\circ} & 0 & q_{N-1}^{\circ} & 0 & q_{N-3}^{\circ} & \ldots & q_{3}^{\circ} & 0 \\
0 & q_{1}^{\circ} & 0 & q_{N-1}^{\circ} & 0 & \ldots & 0 & q_{3}^{\circ} \\
q_{3}^{\circ} & 0 & q_{1}^{\circ} & 0 & q_{N-1}^{\circ} & \ldots & q_{5}^{\circ} & 0 \\
0 & q_{3}^{\circ} & 0 & q_{1}^{\circ} & 0 & & 0 & q_{5}^{\circ} \\
\vdots & & & & & \ddots & \vdots & \vdots \\
\vdots & & & & & & \ddots & \vdots \\
q_{N-1}^{\circ} & 0 & & & & & q_{1}^{\circ} & 0
\end{array}\right]
$$

The real and imaginary parts of the analytic vector $z=x+i \breve{x}$ are orthogonal, i.e.

$$
\sum_{t=0}^{N-1} x_{t} \breve{x}_{t}=0
$$

Hence $x^{\mathrm{T}} \mathcal{H} x=0$, and taking the transpose throughout gives the desired result. Proof of Lemma 2. To show that $\mathcal{H}$ and $\widetilde{\mathcal{W}}_{j}$ commute, we first define the circular 
shift transform matrix $\mathcal{T}$, given by

$$
\mathcal{T}=\left[\begin{array}{cccccc}
0 & 0 & \cdots & 0 & 0 & 1 \\
1 & 0 & \cdots & 0 & 0 & 0 \\
& \ddots & \ddots & & \vdots & \vdots \\
\vdots & & \ddots & \ddots & \vdots & \vdots \\
0 & 0 & \cdots & 1 & 0 & 0 \\
0 & 0 & \cdots & 0 & 1 & 0
\end{array}\right] .
$$

Then with $x=\left(x_{0}, \ldots, x_{N-1}\right)^{\mathrm{T}}$ we have $\mathcal{T} x=\left(x_{N-1}, x_{0}, \ldots, x_{N-2}\right)^{\mathrm{T}}$. Now $\mathcal{T}^{2} \equiv \mathcal{T} \mathcal{T}$, etc., and we can write $\mathcal{H}$ in (A2.3) as $\mathcal{H}=\sum_{n=0}^{N-1} q_{n}^{\circ} \mathcal{T}^{n}$, where $q_{n}^{\circ}=0$ for $n$ even. Similarly $\widetilde{\mathcal{W}}_{j}$ in $(2.1)$ can be written as $\widetilde{\mathcal{W}}_{j}=\sum_{t=0}^{N-1} \tilde{h}_{j, t}^{\circ} \mathcal{T}^{t}$. Hence

$$
\mathcal{H} \widetilde{\mathcal{W}}_{j}=\sum_{n=0}^{N-1} \sum_{t=0}^{N-1} q_{n}^{\circ} \tilde{h}_{j, t}^{\circ} \mathcal{T}^{n+t}=\sum_{t=0}^{N-1} \sum_{n=0}^{N-1} \tilde{h}_{j, t}^{\circ} q_{n}^{\circ} \mathcal{T}^{t+n}=\widetilde{\mathcal{W}}_{j} \mathcal{H}
$$

as required.

Proof of Lemma 3. Since $\breve{\epsilon}_{t}=\sum_{l=0}^{N-1} q_{l}^{\circ} \epsilon_{(t-l) \bmod N}$ we can represent $\breve{\epsilon}_{t}$ through an inverse discrete Fourier transform,

$$
\breve{\epsilon}_{t}=\frac{1}{N} \sum_{k=0}^{N-1} Q_{k} \Xi_{k} e^{i 2 \pi t k / N}
$$

where $Q_{k}=\sum_{l=0}^{N-1} q_{l}^{\circ} e^{-i 2 \pi k l / N}=-i \operatorname{sgnd}_{k}$, the discrete sign sequence $\operatorname{sgnd}_{k}$ is defined in Appendix 1, equation (A1.3), and $\Xi_{k}=\sum_{t=0}^{N-1} \epsilon_{t} e^{-i 2 \pi k t / N}$. Hence,

$$
\begin{aligned}
E\left(\breve{\epsilon}_{t} \breve{\epsilon}_{s}\right)=E\left(\breve{\epsilon}_{t} \breve{\epsilon}_{s}^{*}\right) & =E\left(\frac{1}{N^{2}} \sum_{k=0}^{N-1} Q_{k} \Xi_{k} e^{i 2 \pi t k / N} \sum_{l=0}^{N-1} Q_{l}^{*} \Xi_{l}^{*} e^{-i 2 \pi s l / N}\right) \\
& =\frac{1}{N^{2}} \sum_{k=0}^{N-1} \sum_{l=0}^{N-1} Q_{k} Q_{l}^{*} E\left(\Xi_{k} \Xi_{l}^{*}\right) e^{i 2 \pi(t k-s l) / N},
\end{aligned}
$$

where $Q_{l}^{*}$ is the complex conjugate of $Q_{l}$. However,

$$
E\left(\Xi_{k} \Xi_{l}^{*}\right)=\sum_{u=0}^{N-1} \sum_{v=0}^{N-1} E\left(\epsilon_{u} \epsilon_{v}^{*}\right) e^{-i 2 \pi(u k-v l) / N}=\sigma_{\epsilon}^{2} \sum_{u=0}^{N-1} e^{-i 2 \pi u(k-l) / N}=N \sigma_{\epsilon}^{2} \delta_{k, l},
$$


where $\delta_{k, l}$ is the Kronecker delta. Hence, since $Q_{0}=Q_{N / 2}=0$,

$$
\begin{aligned}
E\left(\breve{\epsilon}_{t} \breve{\epsilon}_{s}\right) & =\frac{1}{N^{2}} \sum_{k=0}^{N-1} \sum_{l=0}^{N-1} Q_{k} Q_{l}^{*} N \sigma_{\epsilon}^{2} \delta_{k, l} e^{i 2 \pi(t k-s l) / N}=\frac{\sigma_{\epsilon}^{2}}{N} \sum_{k=0}^{N-1}\left|Q_{k}\right|^{2} e^{i 2 \pi k(t-s) / N} \\
& =\frac{\sigma_{\epsilon}^{2}}{N}\left(\sum_{k=1}^{\frac{N}{2}-1} e^{i 2 \pi k(t-s) / N}+\sum_{k=\frac{N}{2}+1}^{N-1} e^{i 2 \pi k(t-s) / N}\right) \\
& =\frac{\sigma_{\epsilon}^{2}}{N}\left(\sum_{k=0}^{N-1} e^{i 2 \pi k(t-s) / N}-1-e^{i \pi(t-s)}\right) .
\end{aligned}
$$

Therefore,

$$
E\left(\breve{\epsilon}_{t} \breve{\epsilon}_{s}\right)= \begin{cases}\sigma_{\epsilon}^{2} \frac{N-2}{N}, & \text { if } s=t \\ -\sigma_{\epsilon}^{2} \frac{\left[1+(-1)^{t-s}\right]}{N}, & \text { otherwise }\end{cases}
$$

from which the stated lemma follows.

Proof of Theorem 1. Consider first the stationary wavelet transform coefficients of the analytic vector, $z^{\phi}$ say, for the phase-shifted signal $x^{\phi}$, when we use real-valued level- $j$ periodised wavelet filters $\left\{\tilde{h}_{j, l}^{\circ}\right\}$. Since $\widetilde{W}_{j, t}^{(\phi)}=\sum_{l=0}^{N-1} \tilde{h}_{j, l}^{\circ} z_{(t-l) \bmod N}^{\phi}$,

$$
\left|\widetilde{W}_{j, t}^{\left(z^{\phi}\right)}\right|^{2}=\left|\frac{1}{N} \sum_{k=0}^{N-1} \widetilde{H}_{j}\left(\frac{k}{N}\right) Z_{k}^{\phi} e^{i 2 \pi t k / N}\right|^{2},
$$

where $\widetilde{H}_{j}\left(\frac{k}{N}\right)=\sum_{l=0}^{L_{j}-1} \tilde{h}_{j, l} e^{-i 2 \pi l k / N}$ and $Z_{k}^{\phi}=\sum_{t=0}^{N-1} z_{t}^{\phi} e^{-i 2 \pi t k / N}$. The assumption that the wavelet filter has no energy at zero frequency implies that $\widetilde{H}_{j}(0)=0$, and the assumption that the data have no energy at the Nyquist frequency gives $X_{N / 2}=0 \Rightarrow Z_{N / 2}^{\phi}=0$. Furthermore, since $z^{\phi}$ is the analytic vector for $x^{\phi}$ we know that $Z_{k}^{\phi}=2 X_{k} e^{i \phi}, k=1, \ldots,(N / 2)-1$ and $Z_{k}^{\phi}=0, k=(N / 2)+1, \ldots, N-1$. Hence

$$
\begin{aligned}
\left|\widetilde{W}_{j, t}^{\left(z^{\phi}\right)}\right|^{2} & =\left|\sum_{k=1}^{(N / 2)-1} \widetilde{H}_{j}\left(\frac{k}{N}\right) Z_{k}^{\phi} e^{i 2 \pi t k / N}\right|^{2} \\
& =\sum_{k=1}^{(N / 2)-1} \sum_{l=1}^{(N / 2)-1} \widetilde{H}_{j}\left(\frac{k}{N}\right) \widetilde{H}_{j}^{*}\left(\frac{l}{N}\right) Z_{k}^{\phi}\left(Z_{l}^{\phi}\right)^{*}
\end{aligned}
$$

However $Z_{k}^{\phi}\left(Z_{l}^{\phi}\right)^{*}=4 X_{k} X_{l}^{*}$, and so does not depend on $\phi$, as required. Hence we have proved part (i) of the theorem. 
Now consider the stationary wavelet transform coefficients for the phase-shifted signal $x^{\phi}$, when we use complex-valued level- $j$ periodised wavelet filters $\left\{\tilde{c}_{j, l}^{\circ}\right\}$, say. Since $\widetilde{W}_{j, t}^{\left(x^{\phi}\right)}=\sum_{l=0}^{N-1} \tilde{c}_{j, l}^{\circ} x_{(t-l) \bmod N}^{\phi}$

$$
\left|\widetilde{W}_{j, t}^{\left(x^{\phi}\right)}\right|^{2}=\left|\frac{1}{N} \sum_{k=0}^{N-1} \widetilde{C}_{j}\left(\frac{k}{N}\right) X_{k}^{\phi} e^{i 2 \pi t k / N}\right|^{2}
$$

where $\widetilde{C}_{j}\left(\frac{k}{N}\right)=\sum_{l=0}^{L_{j}-1} \tilde{c}_{j, l} e^{-i 2 \pi l k / N}$ and $X_{k}^{\phi}=\sum_{t=0}^{N-1} x_{t}^{\phi} e^{-i 2 \pi t k / N}$.

The assumption that the wavelet filter has no energy at zero frequency implies that $\widetilde{C}_{j}(0)=0$, and the assumption that the data have no energy at the Nyquist frequency gives $X_{N / 2}=0 \Rightarrow X_{N / 2}^{\phi}=0$. Hence

$$
\left|\widetilde{W}_{j, t}^{\left(x^{\phi}\right)}\right|^{2}=\left|\sum_{k=1}^{(N / 2)-1} \widetilde{C}_{j}\left(\frac{k}{N}\right) X_{k}^{\phi} e^{i 2 \pi t k / N}+\sum_{k=(N / 2)+1}^{N-1} \widetilde{C}_{j}\left(\frac{k}{N}\right) X_{k}^{\phi} e^{i 2 \pi t k / N}\right|^{2}
$$

For a phase shift of $\phi$, we know that $X_{k}^{\phi}=X_{k} e^{i \phi}$ for $k=1, \ldots,(N / 2)-1$ and $X_{k}^{\phi}=X_{k} e^{-i \phi}$ for $k=(N / 2)+1, \ldots, N-1$. Therefore,

$$
\begin{aligned}
\left|\widetilde{W}_{j, t}^{\left(x^{\phi}\right)}\right|^{2} & =\sum_{k, l=1}^{(N / 2)-1} \widetilde{C}_{j}\left(\frac{k}{N}\right) \widetilde{C}_{j}^{*}\left(\frac{l}{N}\right) X_{k} X_{l}^{*} e^{-2 i \pi t(l-k) / N} \\
& +e^{i 2 \phi} \sum_{k, l=1}^{(N / 2)-1} \widetilde{C}_{j}\left(\frac{k}{N}\right) \widetilde{C}_{j}^{*}\left(\frac{(N / 2)+l}{N}\right) X_{k} X_{N / 2+l}^{*} e^{-i 2 \pi t((1 / 2)+(l-k) / N)} \\
& +e^{-i 2 \phi} \sum_{k, l=1}^{(N / 2)-1} \widetilde{C}_{j}\left(\frac{(N / 2)+k}{N}\right) \widetilde{C}_{j}^{*}\left(\frac{l}{N}\right) X_{N / 2+k} X_{l}^{*} e^{-2 i \pi t(-(1 / 2)+(l-k) / N)} \\
& +\sum_{k, l=(N / 2)+1}^{N-1} \widetilde{C}_{j}\left(\frac{k}{N}\right) \widetilde{C}_{j}^{*}\left(\frac{l}{N}\right) X_{k} X_{l}^{*} e^{-i 2 \pi t(l-k) / N}
\end{aligned}
$$

Then, as $x_{t} \in \mathbb{R}$, the Fourier coefficients of the signal are Hermitian symmetric 
and so:

$$
\begin{aligned}
& \left|\widetilde{W}_{j, t}^{\left(x^{\phi}\right)}\right|^{2}=\left|\sum_{k=1}^{(N / 2)-1} \widetilde{C}_{j}\left(\frac{k}{N}\right) X_{k} e^{i 2 \pi t k / N}\right|^{2}+\left|\sum_{l=(N / 2)+1}^{N-1} \widetilde{C}_{j}\left(\frac{l}{N}\right) X_{l} e^{i 2 \pi t l / N}\right|^{2} . \\
& +e^{i 2 \phi} \sum_{k, l=1}^{(N / 2)-1} \widetilde{C}_{j}\left(\frac{k}{N}\right) \widetilde{C}_{j}^{*}\left(\frac{-l}{N}\right) X_{k} X_{l} e^{-i 2 \pi t((1 / 2)+(l-k) / N)} \\
& +e^{-i 2 \phi} \sum_{k, l=1}^{(N / 2)-1} \widetilde{C}_{j}\left(\frac{-k}{N}\right) \widetilde{C}_{j}^{*}\left(\frac{l}{N}\right) X_{k}^{*} X_{l}^{*} e^{-2 i \pi t(-(1 / 2)+(l-k) / N)} \\
& =\left|\sum_{k=1}^{(N / 2)-1} \widetilde{C}_{j}\left(\frac{k}{N}\right) X_{k} e^{i 2 \pi t k / N}\right|^{2}+\left|\sum_{l=(N / 2)+1}^{N-1} \widetilde{C}_{j}\left(\frac{l}{N}\right) X_{l} e^{i 2 \pi t l / N}\right|^{2} \\
& +2 \sum_{k, l=1}^{N / 2-1} \Re\left\{e^{i 2 \phi} \widetilde{C}_{j}\left(\frac{k}{N}\right) \widetilde{C}_{j}^{*}\left(\frac{-l}{N}\right) X_{k} X_{l}(-1)^{t}\right\} e^{-2 i \pi t(l-k) / N} .
\end{aligned}
$$

Consider as input a single sinusoid $x_{t}=\cos \left(2 \pi k_{0} t / N\right)$ with Fourier frequency $k_{0} / N, k_{0} \neq N / 2$. Then $X_{k}=N / 2$ for $k= \pm k_{0} \bmod N$ and equation (A2.7) reduces to

$$
\left|\widetilde{W}_{j, t}^{\left(x^{\phi}\right)}\right|^{2}=\left|(N / 2) \widetilde{C}_{j}\left(\frac{k_{0}}{N}\right)\right|^{2}+\left|(N / 2) \widetilde{C}_{j}\left(\frac{-k_{0}}{N}\right)\right|^{2}+(-1)^{t}\left(N^{2} / 2\right) \Re\left\{e^{i 2 \phi} \widetilde{C}_{j}\left(\frac{k_{0}}{N}\right) \widetilde{C}_{j}^{*}\left(\frac{-k_{0}}{N}\right)\right\} .
$$

For no dependence on the phase-shift $\phi$ we therefore require, for each $k_{0}$,

$$
\Re\left\{e^{i 2 \phi} \widetilde{C}_{j}\left(\frac{k_{0}}{N}\right) \widetilde{C}_{j}^{*}\left(\frac{-k_{0}}{N}\right)\right\}=0 .
$$

Now write the complex-valued level- $j$ wavelet filter $\left\{\tilde{c}_{j, l}\right\}$ as $\tilde{c}_{j, l}=\tilde{a}_{j, l}+i \tilde{b}_{j, l}$. Then $\widetilde{C}_{j}\left(\frac{k_{0}}{N}\right)=\sum_{l=0}^{L_{j}-1} \tilde{c}_{j, l} e^{-i 2 \pi l k_{0} / N}=\sum_{l=0}^{L_{j}-1}\left(\tilde{a}_{j, l}+i \tilde{b}_{j, l}\right) e^{-i 2 \pi l k_{0} / N}=\widetilde{A}_{j}\left(\frac{k_{0}}{N}\right)+$ $i \widetilde{B}_{j}\left(\frac{k_{0}}{N}\right)$, say. Now write the complex variables $\widetilde{A}_{j}\left(\frac{k_{0}}{N}\right)$ and $\widetilde{B}_{j}\left(\frac{k_{0}}{N}\right)$ in polar form, $\widetilde{A}_{j}\left(\frac{k_{0}}{N}\right)=R_{1} \cos (\varphi)+i R_{1} \sin (\varphi)=r+i s$, say, and $\widetilde{B}_{j}\left(\frac{k_{0}}{N}\right)=R_{2} \cos (\theta)+$ $i R_{2} \sin (\theta)=u+i v$, say. Then

$$
\Re\left\{e^{i 2 \phi} \widetilde{C}_{j}\left(\frac{k_{0}}{N}\right) \widetilde{C}_{j}^{*}\left(\frac{-k_{0}}{N}\right)\right\}=\cos (2 \phi)\left(r^{2}-s^{2}+u^{2}-v^{2}\right)-2 \sin (2 \phi)(r s+u v)=0 .
$$

The resulting requirement that $r^{2}+u^{2}=s^{2}+v^{2}$ means that $R_{1}^{2} \cos ^{2} \varphi+$ $R_{2}^{2} \cos ^{2} \theta=R_{1}^{2} \sin ^{2} \varphi+R_{2}^{2} \sin ^{2} \theta \Rightarrow\left(R_{1}^{2} / R_{2}^{2}\right)=-\cos (2 \theta) / \cos (2 \varphi)$.

The requirement that $r s+u v=0$ means that $R_{1}^{2} \cos \varphi \sin \varphi+R_{2}^{2} \cos \theta \sin \theta=$ $0 \Rightarrow\left(R_{1}^{2} / R_{2}^{2}\right)=-\sin (2 \theta) / \sin (2 \varphi)$. 
Hence $\tan (2 \theta)=\tan (2 \varphi)$, which means that $\theta=\varphi \pm n \pi / 2, n \in \mathbb{N}$, so that $R_{1}^{2} \sin (2 \varphi)=-R_{2}^{2} \sin (2 \varphi \pm n \pi)=R_{2}^{2} \sin (2 \varphi)(-1)^{n+1}$. We hence require that $R_{1}=$ $R_{2}$ and $n$ is odd, corresponding to a phase shift of $\pm \pi / 2$ between $\varphi$ and $\theta$. We can thus see that for invariance to a phase shift $\phi$ we require the frequency responses of the real and imaginary parts of the complex-valued filters to have identical magnitudes and to differ in phase by $\pm \pi / 2$ for all such Fourier frequencies. This completes the proof of part (ii) of the theorem.

\section{REFERENCES}

BRUCE, A. G., GAO, H-Y. \& STUETZLE, W. (1999). Subset-selection and ensemble methods for wavelet de-noising. Statist. Sinica 9, 167-82.

COIFMAN, R. R. \& DONOHO, D. L. (1995). Translation-invariant denoising. In Wavelets and Statistics (Lecture Notes in Statistics, Volume 103), Ed. A. Antoniadis and G. Oppenheim, pp. 125-50. New York: Springer-Verlag.

DONOHO, D. L. \& JOHNSTONE, I. M. (1994). Ideal spatial adaptation by wavelet shrinkage. Biometrika 81, 425-55.

DYKSTRA, R. L. (1980). Product inequalities involving the multivariate normal distribution. J. Am. Statist. Assoc. 75, 646-50.

LAWTON, W. (1993). Applications of complex valued wavelet transforms in subband decomposition. IEEE Trans. Signal Process. 41, 3566-8.

LILLY, J. M. \& PARK, J. (1995). Multiwavelet spectral and polarization analyses of seismic records. Geophys. J. Int. 122, 1001-21.

LINA, J-M. \& MAYRAND, M. (1995). Complex Daubechies wavelets. Appl. Comp. Harmonic Anal. 2, 219-29.

MARPLE, S. L. Jr. (1999). Computing the discrete-time "analytic" signal via FFT. IEEE Trans. Signal Process. 47, 2600-3.

OPPEnheim, A. V. \& SCHAFER, R. W. (1975). Digital Signal Processing. Englewood Cliffs: Prentice-Hall.

PAPOUliS, A. (1991). Probability, Random Variables, and Stochastic Processes, 3rd ed.. New York: McGraw-Hill. 
PERCIVAL, D. B. \& MOFJELD, H. (1997). Analysis of subtidal coastal sea level fluctuations using wavelets. J. Am. Statist. Assoc. 92, 868-80.

PERCIVAL, D. B. \& WALDEN, A. T. (2000). Wavelet Methods for Time Series Analysis. Cambridge: Cambridge University Press.

SARDY, S. (2000). Minimax threshold for denoising complex signals with Waveshrink. IEEE Trans. Signal Process. 48, 1023-8.

WALDEN, A. T. \& CONTRERAS CRISTÁN, A. (1998). Matching pursuit by undecimated discrete wavelet transform for non-stationary time series of arbitrary length. Statist. Comp. 8, 205-19.

WALDEN, A. T. \& WILLIAMS, M. L. (1993). Deconvolution, bandwidth and the trispectrum. J. Am. Statist. Assoc. 88, 1323-9.

WHITE, R. E. (1988). Maximum kurtosis phase correction. Geophys. J. Int. 95, 371-89. 


\begin{tabular}{lccccccc} 
Model & 1 & 2 & 3 & 4 & 5 & 6 & 7 \\
\hline signal-to-noise ratio of 10 & 9.9 & 3.7 & 40.2 & 18.0 & 4.9 & 4.2 & 4.3 \\
signal-to-noise ratio of 50 & 21.7 & 5.9 & 51.7 & 18.1 & 1.9 & 7.8 & 7.4 \\
\hline
\end{tabular}

Table 1: Percentage average estimated mean square error inflation $\bar{\alpha}_{p}$ when using standard stationary wavelet transform thresholding rather than the analytic version for signal-to-noise variance ratios of 10 and 50 . 


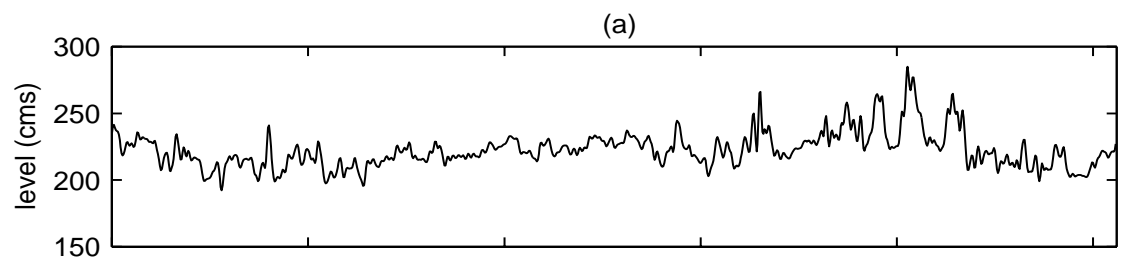

(b)

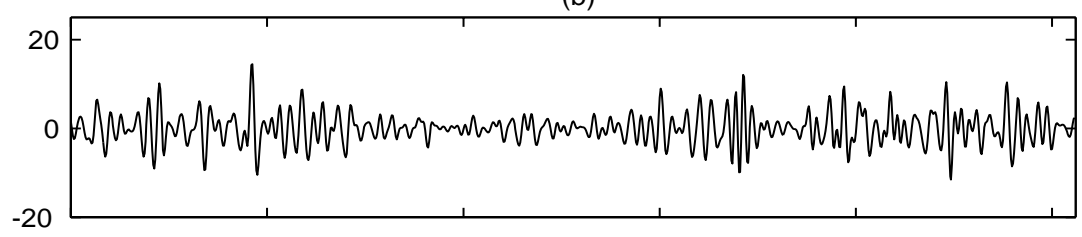

(c)

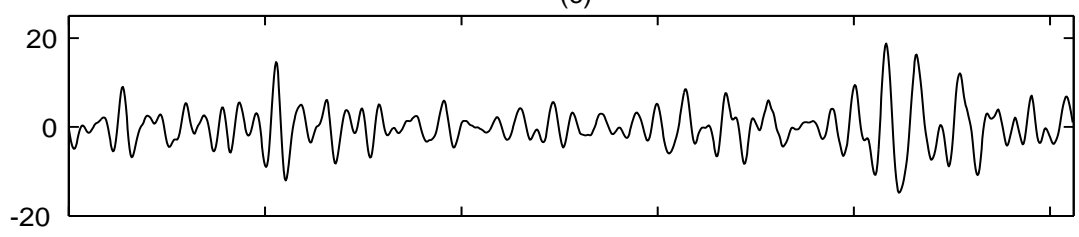

(d)

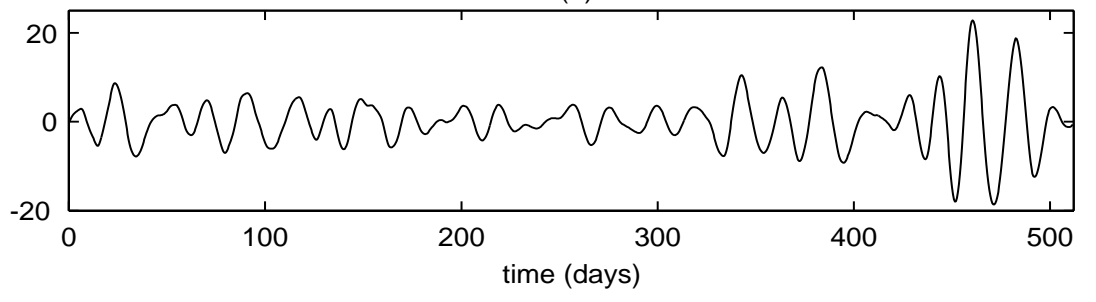

Figure 1: Crescent City, California sea level data: (a) subtidal sea levels in cms. (b)-(d) stationary wavelet transform sequences for levels $j=3$ to 5 , respectively. 

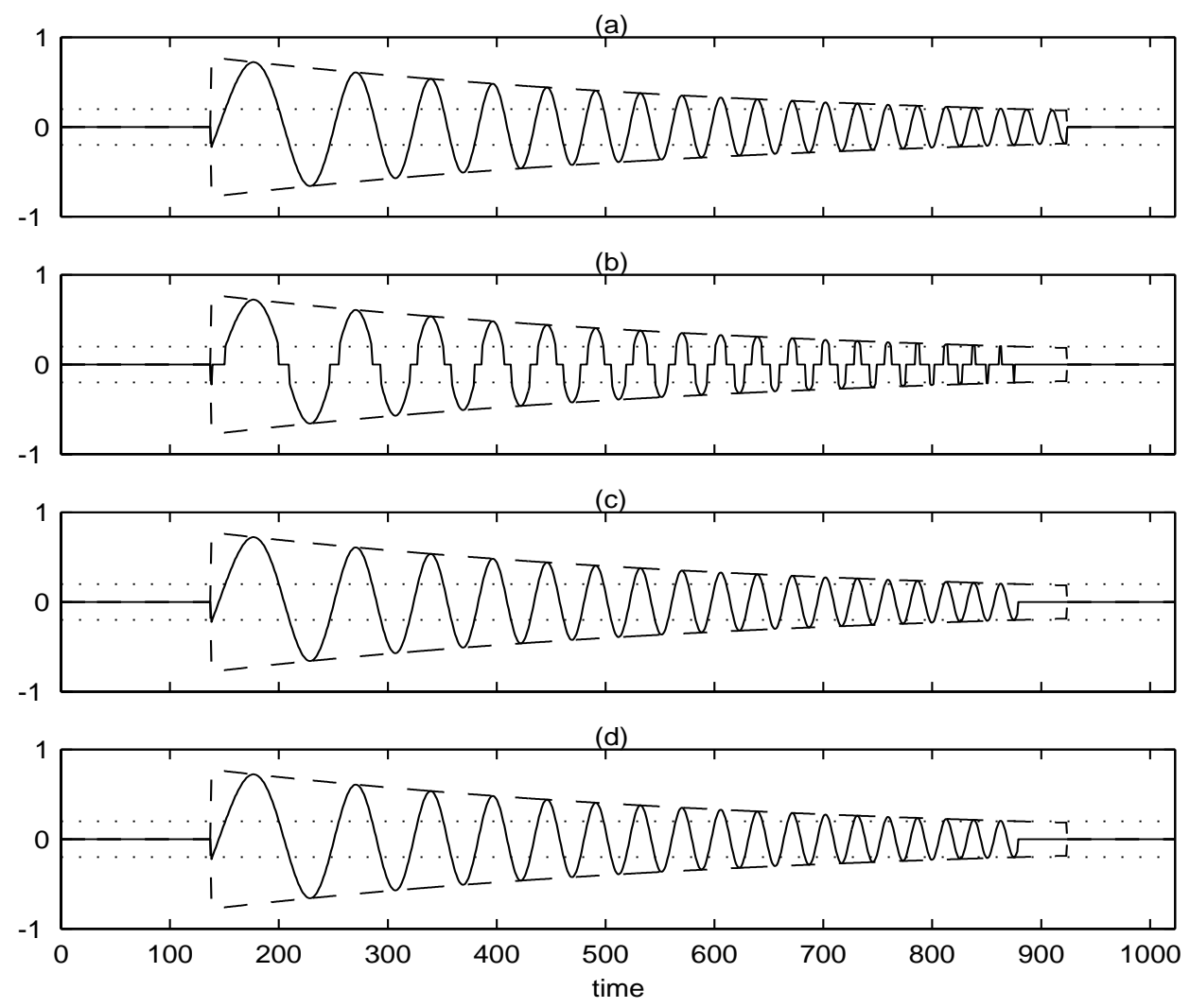

Figure 2: Artificial example. (a) The signal, solid, its envelope, dashed, and the thresholds at \pm 0.2 , dotted. (b) Conventionally thresholded signal. (c) Signal thresholded using the true amplitude $a_{t}$. (d) Signal thresholded using the amplitude from the 'analytic' version of the signal. 


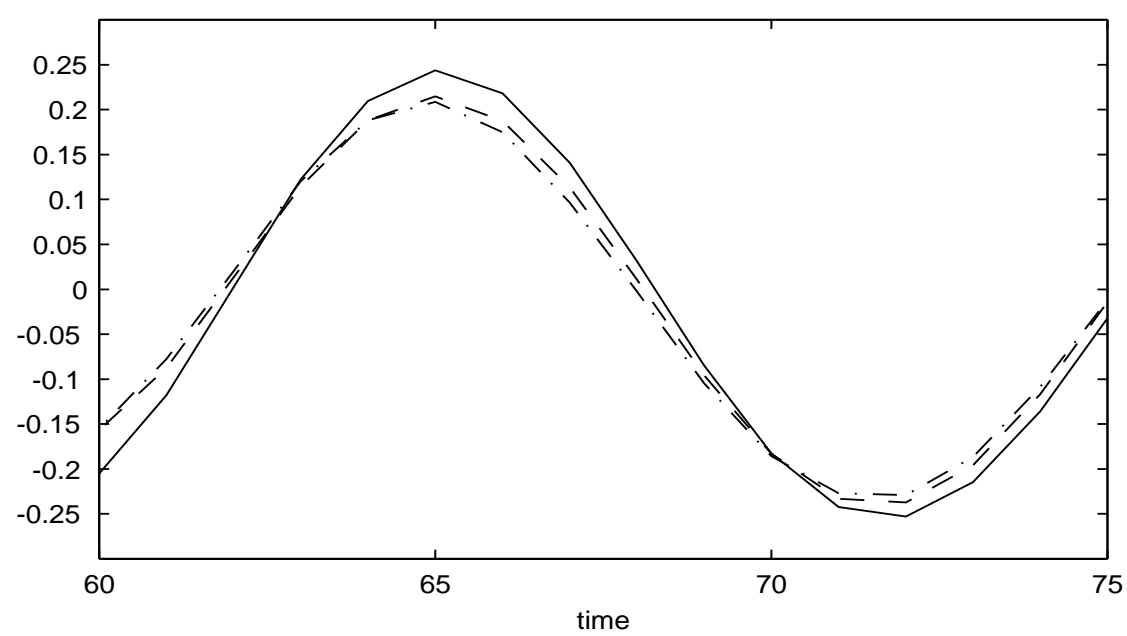

Figure 3: A magnified part of the Doppler signal, solid, and corresponding denoised estimates using the analytic stationary wavelet transform, dashed, and standard stationary wavelet transform, dash-dot. The signal-to-noise variance ratio is 50 . 
(a)

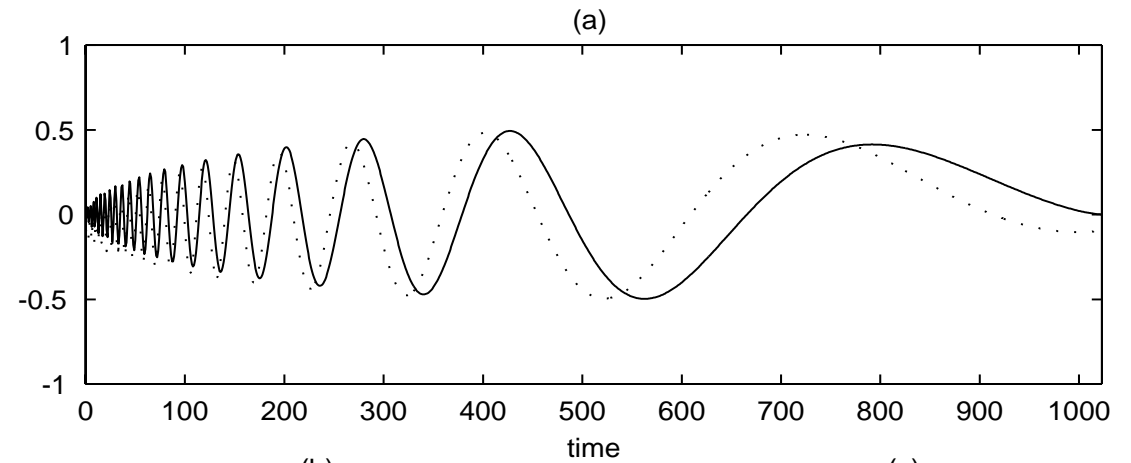

(b)

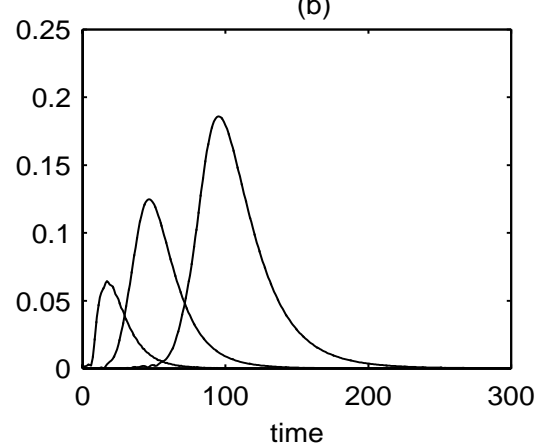

(c)

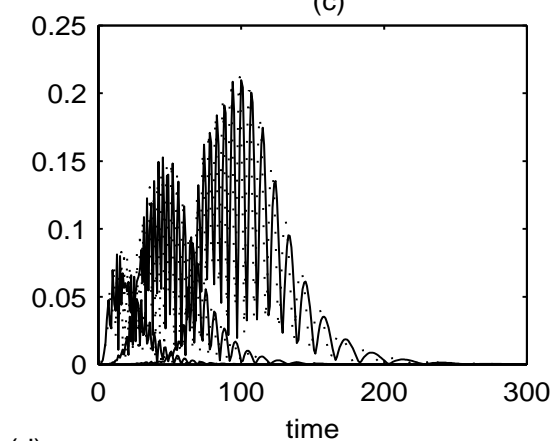

(d)

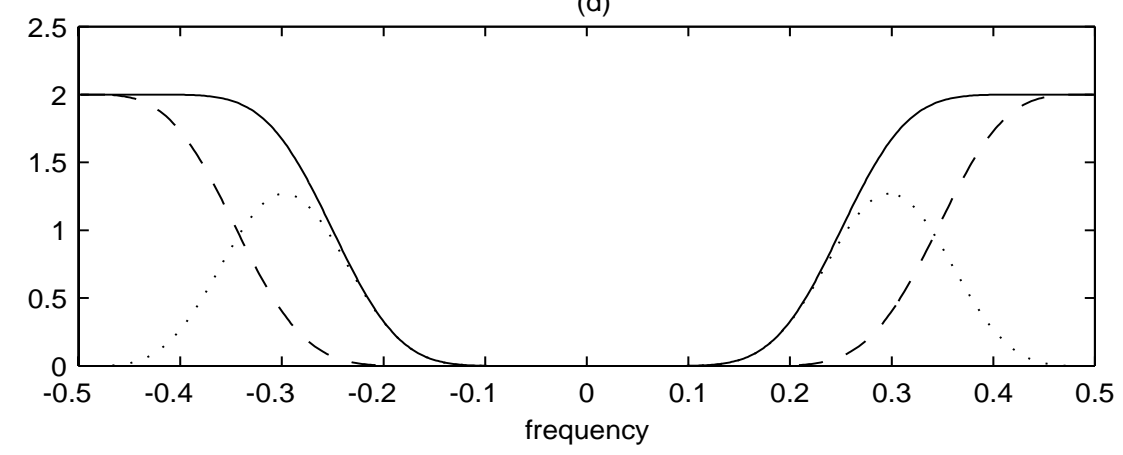

Figure 4: The Doppler signal. (a) The signal, solid, and a $\pi / 4$ phase-shifted version, dotted. (b) Magnitudes of the stationary wavelet transform coefficients of the analytic vector for levels 1 to 3 when using Daubechies extremal phase filters of length 10. (c) Magnitudes of the stationary wavelet transform coefficients of the real vector for levels 1 to 3 when using complex Daubechies wavelet filters of length 10. (d) The modulus squared of the Fourier transform of the complex Daubechies symmetric wavelet filter of length 10 for the complex filter, solid, its real part, dashed, and its imaginary part, dotted. 